\title{
NOTES ON EQUINOX MOTION AND CORRECTIONS TO PRECESSION
}

\author{
T. C. VAN FLANDERN \\ U.S. Naval Observatory, Washington, D.C., U.S.A.
}

(Presented at IAU Colloquium No. 9, 'The IAU System of

Astronomical Constants', Heidelberg, Germany, August 12-14, 1970.)

\section{General}

The coordinate system defined by the locations of distant galaxies should be an inertial coordinate system. Until that system becomes better observed, we must use the stars of our own Galaxy, and make certain assumptions in order to achieve an inertial system. Basically, we assume that the stellar proper motions consist of a component due to galactic rotation (a function of distance from the galactic center), a component due to solar motion, and a random component, peculiar to each star. The random component should not cause the star system as a whole to rotate in any particular direction - in other words, the sum of the random components of proper motion will be zero (to within their standard deviation) in every direction.

The precession of the coordinate system and the proper motions of the individual stars are determined by observing the apparent coordinates at widely spaced epochs. The observed declinations may be regarded as more or less absolute; but the observed right ascensions are relative to some assumed equinox, usually defined by the assigned mean places of a number of catalog stars. These must be revised subsequently, when the true equinox location becomes known from solar or planetary observations.*

When stars are observed fundamentally, we obtain their true declination. However, the right ascensions at any epoch will contain an equinox error, $\Delta E$; and the centennial variations in right ascension will contain an 'equinox motion', $\Delta e$. This latter is simply the result of varying determinations of the equinox error at different epochs.

The problem we then face is the separation of the centennial variations into the parts due to proper motion, and the part due to precession. This may be accomplished by an analysis of the centennial variations in declination, in which solar motion, galactic rotation, and precession are solved for simultaneously. Each has a unique signature.

Starting with a catalog of mean places of stars in which positions and centennial variations of the stars are correctly represented in some system (say the FK4), let us

* There is no information in fundamental observations of star positions alone about where the ecliptic may lie on the celestial sphere. Hence, the equinox location is completely indeterminate from stellar observations alone. Its location must be determined in retrospect from observations of some object which reflects the orbital motion of the Earth relative to the stars. 
examine the effect of changing only the assumed constant of precession, in terms of the precession constants $m$ and $n$

$$
\begin{aligned}
& \Delta \dot{\alpha}=\Delta m+\Delta n \sin \alpha \tan \delta \\
& \Delta \dot{\delta}=\Delta n \cos \alpha
\end{aligned}
$$

where $\dot{\alpha}$ and $\delta$ are the centennial variations. But since the centennial variations are observed quantities, any correction to precession must be compensated by corresponding corrections to the proper motions of the stars

$$
\begin{aligned}
& \Delta \mu_{\alpha}=-\Delta m-\Delta n \sin \alpha \tan \delta \\
& \Delta \mu_{\delta}=-\Delta n \cos \alpha
\end{aligned}
$$

The result of applying these two sets of corrections is that the star positions for any epoch are unchanged, but we have a different separation of the centennial variation into precession and proper motion.

Unlike changing precession, a change in equinox location and motion does affect the observed right ascensions and their centennial variations. The correction is

$$
\begin{aligned}
& \Delta \alpha=\Delta E \\
& \Delta \dot{\alpha}=\Delta e
\end{aligned}
$$

Clearly, the change in $\dot{\alpha}$ must be accomplished by changing $\Delta \mu_{\alpha}$ by an equal amount, rather than by changing the assumed precession constant.

The results of Fricke $(1967 \mathrm{a}, \mathrm{b})$ give corrections to luni-solar precession $\Delta p_{1}$ ranging from +1.08 to $+1 " .38$ per century, with $\Delta m=+1 . .01, \Delta n=+0.44, \Delta e=+1.20$ per century for $\Delta p_{1}=+1$."10. Occultation observations (Martin and Van Flandern, 1970) give $\Delta e=+1.36$ per century. The same observations indicate that $\Delta E=+0.65$ at 1960.0. Both results apply to the FK4 system.

\section{Correcting a Star Catalog}

To correct a star catalog for the effects of equinox motion and a change in the constant of precession, given $\Delta m, \Delta n, \Delta e$, and $\Delta E$ for the catalog epoch, the corrections are

$$
\begin{aligned}
\Delta \alpha & =\Delta E \\
\Delta \delta & =0 \\
\Delta \dot{\alpha} & =\Delta e \\
\Delta \delta & =0 \\
\Delta \mu_{\alpha} & =-\Delta m-\Delta n \sin \alpha \tan \delta+\Delta e \\
\Delta \mu_{\delta} & =-\Delta n \cos \alpha .
\end{aligned}
$$

\section{Correcting Planetary Observations}

Observations of positions of objects in the FK4 system, made relative to FK 4 reference 
stars, should receive the same corrections as the star positions

$$
\begin{aligned}
& \Delta \alpha=\Delta E+\Delta e\left(t-t_{0}\right) \\
& \Delta \delta=0
\end{aligned}
$$

where $t_{0}$ is the epoch of $\Delta E$.

\section{Ephemerides of Sun, Moon, Planets}

When observations of the Sun, Moon, and planets are corrected as described above, their ephemerides require corresponding adjustment if they are to continue to represent the observations. The corrections in $\Delta \lambda, \Delta \beta$ due to $\Delta \alpha($ with $\Delta \delta=0)$ for any position are

$$
\begin{aligned}
& \Delta \lambda=(\cos \varepsilon-\sin \varepsilon \tan \beta \sin \lambda) \Delta \alpha \\
& \Delta \beta=-(\sin \varepsilon \cos \lambda) \Delta \alpha .
\end{aligned}
$$

The resulting implications for ephemerides vary from object to object. The mean longitude at epoch will require the correction $\Delta E\left(\cos \varepsilon-\frac{1}{2} \sin \varepsilon \tan I\right)$, where $I$ is the inclination to the ecliptic. Likewise, the mean motion will increase by $\Delta e\left(\cos \varepsilon-\frac{1}{2}\right.$ $\sin \varepsilon \tan I$ ). The eccentricity and longitude of perihelion (perigee) will both be slightly affected by the presence of the periodic term $-\frac{1}{2} \sin \varepsilon \tan I \sin 2 \lambda$ in longitude. The inclination and nodes will be altered by a somewhat larger amount because of the presence of the latitude term.

\section{Universal and Sidereal Time}

A correction to the equinox of the FK4, changing the right ascensions of all of the catalog stars, has important consequences for the measurement of time. The measures of both sidereal time and Universal time would change by the amount $\Delta E / 15$, according to their present definitions. This would be about 50 to $60 \mathrm{~ms}$ in 1970 . Similarly, the infiuence of $\Delta e$ would be a change in the length-of-day by about $2 \mu \mathrm{s}$.

While these corrections will affect UT2, UTC will of course be unaffected, except insofar as the usual steps may be required to keep UTC within some prescribed tolerance of UT2.

The discontinuity in sidereal time is essential, since the time of transit of the vernal equinox is altered; but that in UT2 is not. The latter could be avoided by altering the expression for the right ascension of the fictitious mean Sun - in other words, by changing the defined relationship between Universal Time and Greenwich Sidereal Time.

\section{Lunar Ephemeris}

The equinox correction to FK4 is quite similar to the equinox difference (NewcombFK4). Hence, the new equinox location will be close to Newcomb's equinox at the present time (but not in Newcomb's time). The lunar theory has Newcomb's equinox for its longitude origin. Hence, little or no correction to the mean longitude of the 
lunar ephemeris $j=2$ is required by the equinox correction. (The mean motion in the lunar ephemeris should be brought onto the same system as the solar ephemeris, in order to eliminate the need for the 1.34 correction to $\Delta T$ as derived from lunar observations.)

\section{Solar Ephemeris and Ephemeris Time}

Since the Sun always lies on the true ecliptic of date, its situation is unique in that observations of the solar declination alone give the true longitude of the Sun, measured from the true equinox, directly. Spencer-Jones utilized this fact in his analysis of solar observations (1939), upon which our present definition of Ephemeris Time (ET) is based. As a result, it would be expected that no correction to the Sun's mean longitude would now be required. Observations, however, cast doubt on this position. But whatever the corrections to the solar ephemeris may be, they bear no simple relation to the equinox correction.

The implications of any change in the Sun's mean longitude are profound for ET, unless the definition is changed, since the Sun moves at a rate of 1 arc sec per 24.349 of ET.

\section{References}

Fricke, W.: 1967a, Astron. J. 72, 642.

Fricke, W.: 1967b, Astron. J. 72, 1368.

Martin, C. F. and Van Flandern, T. C.: 1970, Science 168, 246.

Spencer-Jones, H.: 1939, Monthly Notices Roy. Astron. Soc. 99, 541. 